\title{
Tricuspid valve replacement: a five-year appraisal
}

\author{
R. H. B A X T E R ${ }^{1}, W \cdot H$. B A I N, R. J . R A N K IN, \\ M.A. T UR NER, A. E. ESCAROUS, R. M. THOMSON, \\ A.R. L O R I M E R, and T. D. V. L A W R I E \\ The University Department of Cardiology and the Department of Cardiac Surgery \\ and Radiology, Glasgow Royal Infirmary
}

\begin{abstract}
Baxter, R. H., Bain, W. H., Rankin, R. J., Turner, M. A., Escarous, A. E., Thomson, R. M., Lorimer, A. R., and Lawrie, T. D. V. (1975). Thorax, 30, 158-161. Tricuspid valve replacement: a five-year appraisal. Organic disease of the tricuspid valve required surgery in $18 \%$ of all patients undergoing valve replacement over a five-year period. Differentiation of organic from functional disease is difficult and may be made only at open-heart surgery. The results of a series of 56 patients who underwent tricuspid valve replacement are presented; of these, 53 had organic disease. Operative mortality at the end of five years had fallen to $7 \%$ with significant clinical improvement in $79 \%$ of patients, a reduction in cardiothoracic ratio in $81 \%$, and a low incidence of postoperative tricuspid incompetence.
\end{abstract}

Clinical reports of patients with rheumatic heart disease suggest that $10-20 \%$ have organic tricuspid valve involvement (Sinclair-Smith, 1968; Braimbridge et al., 1970). This is less than the prevalence rates of $22-44 \%$ found at necropsy (Kitchin and Turner, 1964). The discrepancy between clinical and necropsy findings may be due to overshadowing features of mitral and aortic valve disease (Braimbridge et al., 1970) and to difficulty in differentiating organic from functional tricuspid incompetence on the basis of clinical, haemodynamic, and angiographic criteria.

According to Starr, Herr, and Wood (1966), this failure in diagnosis of tricuspid valve disease may be as high as $30 \%$. This has potentially serious consequences (Grondin et al., 1967; Pluth and Ellis, 1969) and accordingly the policy of routinely inspecting or palpating the tricuspid valve in all patients undergoing valve replacement surgery has been adopted whenever there has been any preoperative suspicion of tricuspid valve disease or if at operation the right atrium is enlarged. Subsequent criteria for replacement of the tricuspid valve depend on the degree of stenosis or incompetence noted at operation. This report concerns the results of a series of patients undergoing tricuspid valve replacement over a five-year period (1969-73).

'Present address: Medical Division, The Victoria Infirmary, Glasgow, G42 9TY

\section{PATIENTS AND METHODS}

Fifty-six patients (nine male, 47 female) with a mean吕 age of $44 \pm 8$ years underwent tricuspid valve replacement under cardiopulmonary bypass. All patients hadô severe rheumatic valvular disease requiring reparative ${ }^{\exists}$ surgery of two or more valves (Table). In the initial two years of the series Starr-Edwards prosthetic valves were used. Subsequently the Björk-Shiley tilting disc prosthetic valve has been the choice for all valve음 replacements (Turner et al., 1974).

Patients were classified by functional disability (Newó York Heart Association, Criteria Committee, 1964) Ninety-seven per cent were either grade 3 or 4 pre-i operatively in spite of full medical therapy, and only3 $3 \%$ were grade 2 . All had rheumatic heart disease dominated by mitral and/or aortic valve disease.

Cardiomegaly was present in all patients, and radiological evidence of an enlarged right atrium in $75 \%$, pleural effusion in $25 \%$, and a prominent superior vena cava or azygos vein in $20 \%$. Only seven patients were in sinus rhythm and the remain $-N$ ing 49 were in atrial fibrillation. Other electrocardio-N graphic findings were related to the co-existent mitraf and aortic valve disease.

T A B L E

CONCOMITANT VALVE SURGERY PERFORMED IN ADDITION TO TRICUSPID REPLACEMENT

\begin{tabular}{|c|c|}
\hline Associated Valve Procedure & No. of Patients \\
\hline $\begin{array}{l}\text { Mitral replacement } \\
\text { Mitral and aortic replacement } \\
\text { Mitral and/or aortic valvuloplasty or valvotomy }\end{array}$ & $\begin{array}{r}37 \\
13 \\
6\end{array}$ \\
\hline
\end{tabular}


haEMODYNAMIC AND OPERATIVE FINDINGS Preoperative cardiac catheterization was performed in 46 patients. Pulmonary hypertension was present in all (mean systolic pulmonary artery pressure 62 \pm 24 $\mathrm{mmHg}$ ). As regards the tricuspid valve findings, five had evidence of pure tricuspid stenosis from right heart catheterization and this was confirmed when the valve was seen at operation.

Fifty-one patients had predominant tricuspid regurgitation with shortened distorted valve leaflets which also caused a degree of stenosis in nine patients. In 33 of these patients the classical features of severe tricuspid regurgitation, namely hepatic pulsation and a prominent $\mathrm{V}$ wave in the venous pulse with a systolic plateau wave form in the right atrial tracing, were detected. In the remainder these features were absent, inconstant or the subject of disagreement between observers although the valve was found to be regurgitant at operation. In nine patients a tricuspid lesion was totally unsuspected preoperatively on clinical or catheterization findings, being revealed at operation as a palpable thrill or progressive tricuspid incompetence as cardiopulmonary bypass was being discontinued.

Subsequent histological examination of the excised tricuspid valves revealed organic disease in all but three of the 56 cases. The three patients in whom the valve leaflets were regarded as normal had severe dilatation of the valve ring and co-existent pulmonary hypertension and were considered to have gross functional regurgitation.

\section{RESULTS}

Overall mortality fell dramatically over the five years of this series. In 1969, the first year of tricuspid replacements, operative mortality (defined as within one month of operation) was $66 \%$ and late mortality $34 \%$ but by 1973 this had fallen to $7 \%$ and $7.5 \%$ respectively. Mean length of followup for all survivors leaving hospital was $18 \pm 13$ months. We have compared the results of the 22 patients with tricuspid valve replacements using the Starr-Edwards prosthesis during 1969-70 with the later group of 34 patients having a BjörkShiley prosthesis during 1970-73. Figure 1 illustrates the striking reduction in both hospital and late mortality in the two groups which were comparable in regard to mean age, concomitant valve involvement, pulmonary hypertension, mean right atrial pressure, and clinical disability.

The survivors were reviewed at periods ranging from three to 43 months postoperatively. Clinical improvement in many was striking, $79 \%$ showing an improvement by one clinical grade or more (Fig. 2).

The majority of late deaths were due to cardiac failure (eight patients) or presumed dysrhythmias

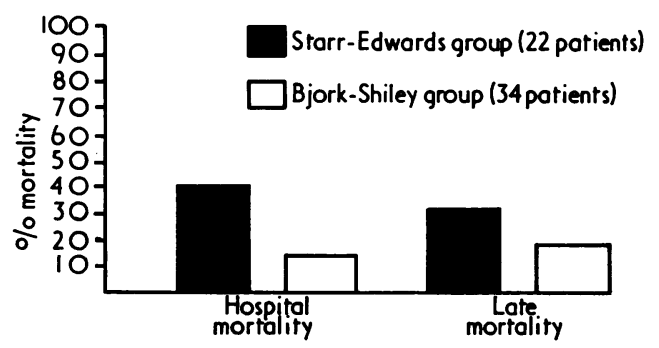

FIG. 1. Comparison of mortality in patients undergoing operation in 1969-70 with a Starr-Edwards prosthesis and in 1970-1973 with a Björk-Shiley prosthesis.

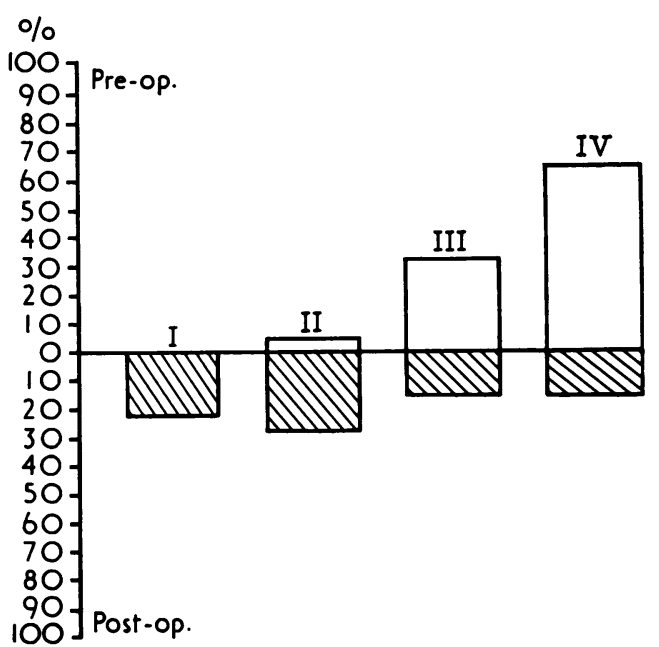

FIG. 2. Pre- and postoperative clinical grading (New York Heart Association criteria).

(three patients) but one patient had pulmonary thromboembolic disease and one an acute viral illness. Of the late deaths, one patient (Fig. 3, patient A), who developed cardiac failure with elevation of the mean right atrial pressure following double valve replacement, died during a second operation to repair a mitral prosthetic leak. At operation excess fibrin deposition was noted on the tricuspid prosthesis but this did not appear to interfere with its function.

Postoperative assessment of the tricuspid valve was made clinically in all patients and by right atrial catheterization in 14. A further patient (Fig. 3, patient B) remained in congestive cardiac failure in spite of triple valve surgery, and this was accompanied by gross elevation of the mean right atrial pressure. In the remainder there was 


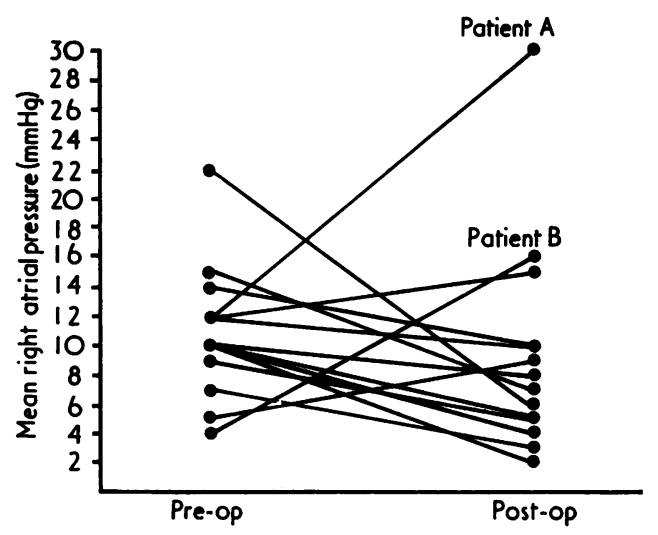

FIG. 3. Pre-and postoperative mean right atrial pressures (14 patients).

no clinical evidence of tricuspid incompetence and all but two showed a fall in mean right atrial pressure.

RADIOLOGICAL FINDINGS Supportive radiological evidence on standard postero-anterior chest radiographs of tricuspid valve disease such as an enlarged right atrium, pleural effusions, and prominent azygos veins and superior venae cavae was inconstant and unrelated to the mean right atrial pressure. A comparison of the pre and postoperative cardiothoracic ratio at the time of follow-up showed a reduction in cardiothoracic ratio in $81 \%$. In addition the absolute cardiac diameter was examined critically and a change in this measurement was regarded as significant only when the absolute transverse diameter varied by more than $0.5 \mathrm{~cm}$ in several successive comparable films. Using this more strict criterion, $57 \%$ had a reduction in the absolute transverse cardiac diameter postoperatively. This reduction correlated well with clinical improvement in that patients who failed to improve or deteriorated showed insignificant changes or increased cardiac diameters respectively.

\section{DISCUSSION}

The variation in the frequency of tricuspid valve disease in rheumatic heart disease has been emphasized in previous studies. Thus $15-21 \%$ of patients at mitral valvotomy (Bailey and Bolton, 1956; Kitchin and Turner, 1964; Starr et al., 1966) or double valve replacement (Starr et al., 1966) have significant tricuspid pathology.

The current series of 56 patients represented $18 \%$ of all patients undergoing mitral and/or aortic valve replacement during the same five $\vec{\Rightarrow}$ year period.

The accurate differentiation of organic from functional incompetence can be difficult by clini cal evaluation or even by catheterization anfक्ष angiography, and attempts to quantify the degree of incompetence are unreliable (Cairns et al. \$) 1968). As a result, inspection and assessment at the time of operation become essential (Starr ett al., 1966; Pluth and Ellis, 1969; Molloy andw Clelland, 1972).

The management of tricuspid regurgitation hask been controversial, opinion being divided on the necessity for surgical treatment in organic aș compared to functional regurgitation and between valvuloplasty and valve replacement as the opera $\infty$ tion of choice. High mortality and poor functionat results in untreated series (Grondin et al., 1967) can be contrasted with that reported by Brauno wald, Ross, and Morrow (1967), who found that: patients with tricuspid incompetence undergoingo mitral valve replacement made slower postoperaer tive progress but that eventually spontaneous reo gression of the tricuspid regurgitation occurred? Other workers (Pluth and Ellis, 1969; Starr et al. 1966) have not confirmed this spontaneous regresa sion of tricuspid regurgitation though it may bळ that this is due to confusion in definition of organic and functional disease.

Experience in this centre is that many patients? in whom tricuspid valve replacement was con sidered at operation but not proceeded with: deteriorated in the postoperative period with pro gressive signs of tricuspid regurgitation. Thre such patients required readmission for tricuspif valve replacement within 18 months of the first operation (a mitral valve replacement).

Overall it seems that organic regurgitation due to thickened, shortened, and adherent valve leaf lets, and often accompanied by stenosis, should be treated by valve replacement. While some patients with mild to moderate functional regurgftation will regress with correction of mitral and $\%$ or aortic valve dysfunction, there are those in whom functional regurgitation is gross and reo quires either a valve replacement or valvuloplast (Pluth and Ellis, 1969; Starr, 1969).

Much of the dramatic symptomatic and haem\& dynamic improvement in these patients is du跣 to the operative correction of co-existent valve dysfunction but it is noted that the excellent funcs tional result and the low incidence of postoperat tive tricuspid regurgitation recorded in this an other series of tricuspid valve replacement were not found in a comparable series when untreated 
or subjected to valvuloplasty by Pluth and Ellis (1969).

An operative mortality of $25 \%$ has been recorded in previous series though Starr's achievement of $6 \%$ for combined mitral and tricuspid replacements is surprising when compared with the $15 \%$ mortality for pure mitral valve replacement. The extremely high operative mortality in the initial years of this study has progressively fallen annually to the acceptable level of $7 \%$ during the last year. There are several possible reasons for this but the most important seems likely to be the continuing improvement in surgical and postoperative techniques coupled with the use of a low-profile, low-inertia prosthesis.

\section{REFERENCES}

Bailey, C. P. and Bolton, H. E. (1956). Criteria for and results of surgery for mitral stenosis. New York State Journal of Medicine, 56, 649.

Braimbridge, M. V., Sarin, C. L., Yalav, E., Clement, A. J., and Mendel, D. (1970). Tricuspid valve replacement in rheumatic heart disease. British Medical Journal, 3, 629.

Braunwald, N. S., Ross, J., and Morrow, A. G. (1967). Conservative management of tricuspid regurgitation in patients undergoing mitral valve replacement. Circulation, 35, Supplement $1,63$.

Cairns, K. B., Kloster, F. E., Bristow, J. D., Lees, M. H., and Griswold, M. E. (1968). Problems in the hemodynamic diagnosis of tricuspid insufficiency. American Heart Journal, 75, 173.
Grondin, P., Lepage, G., Castonguay, Y., and Meere, C. (1967). The tricuspid valve-a surgical challenge. Journal of Thoracic and Cardiovascular Surgery, 53, 7.

Kitchin, A. and Turner, R. (1964). Diagnosis and treatment of tricuspid stenosis. British Heart Journal, 26, 354.

Molloy, P. J. and Clelland, J. (1972). Diagnosis of organic tricuspid valve disease (abstract). British Heart Journal, 34, 208.

New York Heart Association, Criteria Committee (1964). Disease of the Heart and Blood Vessels. Nomenclature and Criteria for Diagnosis, 6th edition, p. 144. Little, Brown, Boston and Churchill, London.

Pluth, J. R. and Ellis, F. H. (1969). Tricuspid insufficiency in patients undergoing mitral valve replacement. Journal of Thoracic and Cardiovascular Surgery, 58, 484.

Sinclair-Smith, B. C. (1968). Diseases of the tricuspid valve. In Cardiovascular Disorders, Ch. 48, pp. 626-638. Davis, Philadelphia.

Starr, A. (1969). Discussion on tricuspid insufficiency in patients undergoing mitral valve replacement, by J. R. Pluth and F. H. Ellis. Journal of Thoracic and Cardiovascular Surgery, 58, 489.

Herr, R., and Wood, J. (1966). Tricuspid replacement for acquired valve disease. Surgery, Gynaecology and Obstetrics, 122, 1295.

Turner, M. A., Bain, W. H., Thomson, R. M. T., Lorimer, A. R., and Lawrie, T. D. V. (1974). Early results of heart valve replacement using Bjork-Shiley prosthesis. Scandinavian Journal of Thoracic and Cardiovascular Surgery (in press).

Requests for reprints to: Dr. R. H. Baxter, Medical Division, The Victoria Infirmary, Glasgow G42 9TY. 